\title{
LV-PP-3-3
}

\section{Immediate outcomes of percutaneous radiofrequency-assisted liver partition and portal vein embolization for staged hepatectomy (PRALPPS) in patients with intrahepatic cholangiocarcinoma (IHCC)}

\author{
Natalya KULIKOVA ${ }^{* 1}$, Yulia KULEZNEVA² ${ }^{2}$ Olga MELEKHINA ${ }^{2}$, Ruslan ALIKHANOV ${ }^{1}$, Mikhail EFANOV \\ 'Department of Hepatobiliary and Pancreatic Surgery, Loginov A.S. Moscow Clinical Scientific Center, Russia \\ ${ }^{2}$ Department of Interventional Radiology, Loginov A.S. Moscow Clinical Scientific Center, Russia
}

Introduction: Surgical resection in intrahepatic cholangiocarcinoma (IHCC) is the only feasible modality with a curative ability. The challenge balances between growing the future liver remnant (FLR) in a short time and the same time preventing tumor progression and surgical complications. Associating liver partition and portal vein ligation for staged hepatectomy (ALPPS) gives a chance for fast kinetic growth, but with high morbidity and mortality

Methods: This observation includes 8 comparative patients with IHCC: 4 underwent portal vein embolization (PVE) and 4, ALPPS (3, RALPPS). PVE group has median age 62.2 years, median FLR volume was $564.4 \mathrm{~mL}, 33 \%$. ALPPS group has median age 58.6 years, median FLR volume was $542.6 \mathrm{~mL}, 34.9 \%$. One patient had classic ALPPS and 3 had mini-invasive variant.

Results: The first stage was uncomplicated in the both groups. The median FLR volume became $785 \mathrm{~mL}(46 \%)$ in a 27.5 days and $753.9 \mathrm{~mL}(38 \%)$ in a 17.7 days in PVE and ALPPS groups respectively. Nevertheless, degree of hypertrophy was similar: $41 \%$ in PVE and $40.4 \%$ in ALPPS. Kinetic growth rate was significantly different: $1.38 \%$ and $2.62 \%$ /day, respectively. Seven patients completed the second stage. Sever morbidity was revealed with only ALPPS group: grade IIIa $(\mathrm{n}=1)$ and grade V $(\mathrm{n}=1)$ according to Clavien-Dindo. The in-hospital death was associated with classical ALPPS in one patient with extremely small FLR $(<30 \%)$.

Conclusions: The modified ALPPS (PRALPPS) may be considered as a safe tool to achieve rapid and sufficient hypertrophy in patients with IHCC. 